\title{
Preparation of meteorological input for AERMOD using Malaysian meteorological data
}

\begin{abstract}
Gaussian plume dispersion model - AMS/EPA Regulatory Model (AERMOD) has been recognised as the preferred regulatory air dispersion model and has been proven to perform better than other available models. However, Malaysian meteorological data has limited parameters and the data recorded is inadequate to be used in AERMOD. Currently, processed meteorological data has to be bought from meteorological data service providers located overseas. The processed data does not represent the real conditions experienced at the site accurately. The study involves the identification of missing data in 4 meteorological stations located in Peninsular Malaysia (Cameron Highlands, Subang, Sepang KLIA, and Kuantan), replacement of the missing data and preparation of the data in accordance with the format that AERMOD requires. The study result in a methodology to replace missing data and calculation using bulk formulae which is developed based on certain assumptions that are practical and scientific.
\end{abstract}

